\section{Cardiac surgery risk scoring systems: In quest for the best}

\author{
Milind Hote
}

Over the last 3 decades, there has been a profusion in the number of cardiac surgery risk score systems available (approximately 20 in current adult cardiac surgery literature). ${ }^{1}$ One common factor in these scoring systems is that they have all been proposed from either North America or Europe. ${ }^{1}$ The field of cardiac surgery is continuously evolving with changes in surgical indications, spectrum of diseases, surgical expertise, perioperative management and extensiveness of surgical audit. Consequently, newer scoring systems have been regularly published with the common objective of predicting surgical mortality and more recently, surgical morbidity. Search of literature reveals no scoring system from large population subgroups like Japan, South-east Asia or Africa.

Several reports from these populations have employed the commonly used 'western' risk scoring systems like the European System for Cardiac Operative Risk Evaluation (Euroscore) I, Euroscore II, Parsonnet or the Society of Thoracic Surgeons (STS) systems to their population. There are numerous studies which compare the performance of two or more different scoring systems on some subset of cardiac surgery patients (eg, low risk vs high risk coronary artery bypass grafting (CABG), single/multivalve surgery, $\mathrm{CABG}+$ valve surgery, aortic surgery and so $\mathrm{on}^{2}{ }^{3}$ ). The conclusions commonly drawn indicate that Euroscore II and STS scores are most widely used; however, even these two scores give different predictions in different groups. Thus, these 'western' scores are seen to be relatively 'off-the-mark' in correctly predicting the operative mortality in eastern population, thus essentially being inaccurate to a variable extent ${ }^{4}$. The common 'inaccuracies' reported include variations between the subgroups of elective/semiurgent/emergency surgical procedures and low-risk/ high-risk surgical groups.

These 'discrepancies' raise the following questions:

1. What is the utility of scoring systems? Should all cardiac surgeries at all

Correspondence to Dr Milind Hote, Department of CTVS, All India Institute of Medical Sciences, New Delhi, 110029, India; mphaiims@hotmail.com centres worldwide be performed after MANDATORY assessment and documentation of risk scoring using some specific scoring system?

2. How scoring systems evolved? Why so many scoring systems have come up?

3. Does every country/region need its own scoring system? For those populations (countries) who have not come up with a scoring system till now, which are the best ones to be applied?

To answer the first and second questions, it is pertinent to see the origin, utility and evolution of scoring systems. Scoring systems for cardiac surgery were initially and essentially designed to predict mortality rates after high-risk cardiac surgery. This started because there was a paradigm shift in the manner coronary artery disease was being managed in the 1970s (CABG was the gold standard and the only 'intervention' available) and the 1980s onwards (balloon angioplasty and percutaneous coronary intervention exploded onto the clinical scene). Hence, in the late 1980s, the spectrum of patients undergoing $\mathrm{CABG}$ was rapidly changing (more and more 'high-risk' patients were undergoing CABG surgery). Starting from the Parsonnet score in late $1980 \mathrm{~s}^{5}$, surgeons applied more parameters and variables to derive either more complex or essentially simplified scoring systems and thus came up with Euroscore I, Euroscore II, STS score, Ambler and Age-Creatinine-Ejection Fraction Score and so on. Meanwhile, the paediatric cardiac surgeons also came up with their scoring systems (Risk Adjusted classification for Congenital Heart Surgery-1, Aristotle score, Society of Thoracic Surgery-European Association for Cardio-Thoracic Surgery Mortality Score).

All the scores use various combinations of patient factors like age, comorbid conditions, cardiovascular risk factors, extracardiac risk factors and so on. The evolving scoring systems used either more and more patient parameters or some minimum selected parameters; all designed to hone down to a 'very accurate' prediction model which was not to be. ${ }^{2-4}$ Initial scores were designed by ascribing scores to $\mathrm{n}$ number of designated factors and then subsequent counting of these points to derive the final score for a patient. Today, the systems have evolved to a complex prediction system based on computed statistical analysis of multiple variables using further logistic regression. Still, even today, different scores still give differing prediction of morbidity and mortalities and the parameter used to gauge their accurate predicting abilitythe area under receiver operating characteristic curve and C-index is variable for same patient population utilising different scoring systems. (Good predictive ability is when C-index is above 0.7 and is strong for values above 0.8. $)^{4}$ Scoring systems also evolved because their focus shifted from initial prediction of mortality to predicting major morbidity too. It was realised that predicting only operative mortality did not give the complete picture when trying to envisage the 'cost' of performing a surgical procedure and that morbidity events needed to be also looked into.

The utility of employing risk scores for patients undergoing cardiac surgery is also evolving. Risk scores enable preoperative risk assessment of a patient, thereby explaining operative risk to patients and families. They also enable analysis of costbenefit ratio for a particular operation, thus appropriating resources available with a hospital. Determination of very high-risk score for a particular patient may aid in changing the choice of therapy in such a situation; a good example being the use of transcatheter aortic valve implantation (TAVI) (in place of surgical aortic valve replacement) for an octogenarian patient with severe calcific aortic stenosis. Scoring systems also enable surgical triage (which patient diagnosis to be operated at which centre), quality control-comparison of results of a particular operation between centres (therapy trends). This hospital audit can be helpful in establishing different surgical programmes at different centres (centres of excellence for a particular procedure may be developed, eg, TAVI or minimally invasive cardiac surgery -MICS CABG and so on).

Thus, it is important to have documented risk scoring system for all cardiac surgery patients because it results in the advancement of therapy offered to patients and also enables soft audit of a surgical centre.

There has been no popular scoring system proposed from South east Asia, Japan or Africa. Sino System for Coronary Operative Risk Evaluation ${ }^{6}$ published from China is not in popular use outside China. The third question raised above has been attempted to be answered by several studies in the last 
decade which designate scoring system A or B to be the one most suited for their patient population. ${ }^{2-4} 6$ However, A or B systems also have been shown to be 'off-the-mark' in accurate prediction of perioperative mortality.

The study by Paul Kurlansky et $a l^{7}$ in this issue elegantly raises and discusses this point of limitation (of a well-established scoring system in one population being of limited utility in another major population group having racial and ethnic differences). The authors mention the likely reason for this variation, 'although the underlying physiological substrate undergoing surgery is similar (meaning that physiological characteristics of patients from two population groups are similar), but there is differential interaction and impact of various risk factors to individuals belonging to different racial/ethnic/ geographic/genetic entities'. There could be variable interaction of established risk factors (age, smoking, body mass index, lipid levels) with the genetic constitution of patients in two different populations (eg, Japanese vs Indian subcontinent vs Scandinavian populations have different age profiles for coronary artery disease (CAD) and also the angiographic patterns of disease burden).

Thus, there should be obvious caution in applying one risk score system to another constitutionally different population. This also implies that 'local' risk scoring systems may far better in predicting the perioperative morbidity and mortality statistics and all ethnoracial groups of population perhaps need to come up with their own scoring systems to fulfil this objective prediction. These proposed scoring systems should then be compared and standardised against the more popular 'western' standards with the use of well-designed studies and hence validated over a course of time. This undertaking, however, requires tremendously evolved infrastructural database system and good follow-up regime which are ominously lacking in many 'eastern' countries like India, SAARC countries and African nations.

Rapid advancements are occurring in the field of genetic research and in deciphering molecular mechanisms of diseases like CAD; these advances far outpace the developments in surgical techniques. These basic sciences will enable understanding of the complex interplay between visible patient risk factors and occurrence of actual clinical disease which are different for different populations. Till such time as the less developed countries shore up their database, infrastructural facilities, the east has to adopt the standard scoring systems from the west so as to enable an objective evaluation of their evolving surgical practices. The weight of current literature suggests that Euroscore II and the STS scoring systems suffice this 'standard system' requirement.

Funding The authors have not declared a specific grant for this research from any funding agency in the public, commercial or not-for-profit sectors.

Competing interests None declared.

Patient consent Not required.

Provenance and peer review Commissioned; internally peer reviewed.
(C) Article author(s) (or their employer(s) unless otherwise stated in the text of the article) 2018. All rights reserved. No commercial use is permitted unless otherwise expressly granted.

\section{Check for updates}

To cite Hote M. Heart Asia 2018;10:e011017. doi:10.1136/heartasia-2018-011017

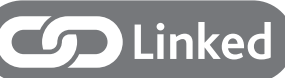

http://dx.doi.org/10.1136/heartasia-2017-010995

Heart Asia 2018;10:e011017. doi:10.1136/

heartasia-2018-011017

\section{REFERENCES}

1 Prins C, de Villiers Jonker I, Botes L, et al. Cardiac surgery risk-stratification models. Cardiovasc J Afr 2012;23:160-4.

2 Geissler HJ, Hölzl P, Marohl S, et al. Risk stratification in heart surgery: comparison of six score systems. Eur J Cardiothorac Surg 2000;17:400-6.

3 Barmettler $\mathrm{H}$, Immer FF, Berdat PA, et al. Riskstratification in thoracic aortic surgery: should the EuroSCORE be modified? Eur I Cardiothorac Surg 2004;25:691-4

4 Wang C, Tang YF, Zhang JJ, et al. Comparison of four risk scores for in-hospital mortality in patients undergoing heart valve surgery: a multicenter study in a Chinese population. Heart Lung 2016;45:423-8.

5 Parsonnet $V$, Dean D, Bernstein AD. A method of uniform stratification of risk for evaluating the results of surgery in acquired adult heart disease. Circulation 1989;79:13-12.

6 Zheng Z, Zhang L, Li X, et al. SinoSCORE: a logistically derived additive prediction model for post-coronary artery bypass grafting in-hospital mortality in a Chinese population. Front Med 2013;7:477-85.

7 Soo-Hoo S, Nemeth S, Baser O, et al. East meets West: the influence of racial, ethnic and cultural risk factors on cardiac surgical risk model performance. Heart Asia 2018;10:e010995. 